\title{
Numbers with a large prime factor
}

\author{
by \\ R. C. Baker (Provo, Ut.) and G. Harman (Cardiff)
}

1. Introduction. Given a large positive number $x$, let $P(x)$ denote the greatest prime factor of

$$
\prod_{x<n \leq x+x^{1 / 2}} n
$$

Lower bounds for $P(x)$ have been given by Ramachandra [15,16], Graham [9], Baker [1], Jia [12] and Liu [13]. The last paper contains the bound

$$
P(x)>x^{0.723} \text {. }
$$

In the present paper we give a sharper bound.

THEOREM 1. For sufficiently large $x$, we have

$$
P(x)>x^{0.732} \text {. }
$$

As in previous papers on the subject, we combine sieve methods with estimates for exponential sums

$$
\sum_{h \leq H} \sum_{m} a_{m} \sum_{v<m n \leq e v} b_{n} e\left(\frac{h x}{m n}\right) .
$$

Here $e(\theta)=e^{2 \pi i \theta}$. The paper of Fouvry and Iwaniec [7] was an important step forward in the study of sums (1.1), and one of the results of [7] is used in [13] with a little adaptation; see Lemma 2, below. Some results in [1] are still useful; see Lemmas 2, 3 .

For the special sums (1.1) in which

$$
a_{m}=1,
$$

the best results are due to Liu [13]. He uses different methods for

$$
v<x^{0.6-\varepsilon}
$$

Research of the first author supported in part by a grant from the National Science Foundation. 
and

$$
v \geq x^{0.6-\varepsilon}
$$

Here and below, $\varepsilon$ is a positive number, supposed sufficiently small.

In the present paper, we give a theorem on bilinear exponential sums from which the results of [13] follow in both cases (1.3), (1.4) (Theorem 2 and its corollaries; see Section 3). Theorem 2 has other applications, which will be taken up elsewhere.

Let $y=x^{1 / 2}, L=\log x$, and

$$
N(d)=\sum_{\substack{x<n \leq x+y \\ d \mid n}} 1 .
$$

Let $v$ be a positive number in $\left(x^{1 / 2}, x^{3 / 4}\right]$. We write $v=x^{\theta}$,

$$
\mathcal{A}=\{n: v<n \leq e v, N(n)=1\}, \quad S(\mathcal{A}, z)=|\{n \in \mathcal{A}: p \mid n \Rightarrow p \geq z\}|
$$

where $p$ denotes a prime variable, and

$$
S(\theta)=|\{p: p \in \mathcal{A}\}| \text {. }
$$

Theorem 1 will follow if we establish that

$$
\sum_{x^{0.732}<p \leq P(x)} N(p) \log p>0 .
$$

Just as in [1], $\S 1$, it suffices to prove that

$$
\sum_{d<x^{3 / 5-\varepsilon}} \Lambda(d) N(d)=\left(\frac{3}{5}-\varepsilon\right) y L+O(y)
$$

and

$$
\sum_{x^{3 / 5-\varepsilon}<p \leq x^{0.732}} N(p) \log p<2 y L / 5
$$

in order to establish (1.5). The formula (1.6) is given in [13], but for the sake of completeness we shall deduce it from Theorem 2 (see Corollary 2).

As on p. 229 of [1], (1.7) will follow from the bound

$$
\int_{0.6-\varepsilon}^{0.732} \theta S(\theta) d \theta<\left(\frac{2}{5}-\varepsilon\right) y L^{-1} .
$$

Thus we seek the sharpest attainable upper bounds for $S(\theta)$. As in $[1,12$, 13], we use the Rosser-Iwaniec sieve, at least for $\theta>0.661$. For $\theta \leq 0.661$, we use the alternative sieve procedure developed by Harman [11] and Baker, Harman and Rivat [2] to give sharper bounds for $S(\theta)$. It is here that we gain a sizeable advantage over Liu [13].

Throughout the paper, we suppose that $x>C(\varepsilon)$ and write $\eta=$ $\exp (-3 / \varepsilon), J=\left[v y^{-1} x^{4 \eta}\right]$. Constants implied by $\ll, \gg$ and $O_{\varepsilon}()$ depend 
at most on $\varepsilon$. Constants implied by $O($ ) are absolute. The notations $A \lesssim B$ and $Y \asymp Z$ are abbreviations for $A \leq B(1+O(\varepsilon))$ and $Y \ll Z \ll Y$.

We write

$$
\begin{aligned}
& \psi(\alpha)=\alpha-[\alpha]-1 / 2, \\
& V(\alpha)=\prod_{p<\alpha}\left(1-\frac{1}{p}\right), \\
& G(\alpha)=\exp \left(1-\left(\frac{1}{\alpha}\right) \log \left(\frac{1}{\alpha}\right)\right) \quad(\alpha>0) .
\end{aligned}
$$

We write $m \sim M$ as an abbreviation for $M<m \leq 2 M$. The smallest prime factor of $n$ is written $Q(n)$, with $Q(1)=\infty$.

The first-named author would like to thank the Institute of Advanced Study at Princeton, where the penultimate draft of the paper was prepared.

\section{Exponential sums of form (1.1)}

Lemma 1. Let $\alpha, \alpha_{1}, \alpha_{2}$ be given real numbers such that $\alpha \neq 1$ and $\alpha \alpha_{1} \alpha_{2} \neq 0$. Let $1 \leq M, M_{1}, M_{2} \leq x$. Let $A>0$, and

$$
S=\sum_{m \sim M}\left|\sum_{m_{1} \sim M_{1}} \sum_{m_{2} \sim M_{2}} b_{m_{1}, m_{2}} e\left(A m^{\alpha} m_{1}^{\alpha_{1}} m_{2}^{\alpha_{2}}\right)\right| \quad \text { where }\left|b_{m_{1}, m_{2}}\right| \leq 1 .
$$

Writing $F=A M^{\alpha} M_{1}^{\alpha_{1}} M_{2}^{\alpha_{2}}, L_{1}=\log \left(M M_{1} M_{2}(1+A)\right)$, we have

$$
\begin{aligned}
S \ll & L_{1}^{2}\left(\left(M_{1} M_{2}\right)^{13 / 14} M^{9 / 14} F^{1 / 14}+M_{1} M_{2} M^{2 / 3}\right. \\
& \left.+M_{1} M_{2} M^{13 / 12} F^{-1 / 4}+\left(M_{1} M_{2}\right)^{3 / 4} M+\left(M_{1} M_{2}\right)^{3 / 4} M^{1 / 2} F^{1 / 4}\right) .
\end{aligned}
$$

P r o of. This is a variant of [7], Theorem 3; see [2] for a proof. In [2], the authors included terms $\left(M_{1} M_{2}\right)^{23 / 24} M F^{-1 / 6}$ and $\left(M_{1} M_{2}\right)^{23 / 24} M^{7 / 12} F^{1 / 24}$. These are superfluous, since

$$
\begin{aligned}
& \left(M_{1} M_{2}\right)^{23 / 24} M F^{-1 / 6} \\
& =\left(M_{1} M_{2} M^{13 / 12} F^{-1 / 4}\right)^{4 / 6}\left(M_{1} M_{2} M^{2 / 3}\right)^{1 / 6}\left(M_{1}^{3 / 4} M_{2}^{3 / 4} M\right)^{1 / 6}, \\
& \left(M_{1} M_{2}\right)^{23 / 24} M^{7 / 12} F^{1 / 24} \\
& \quad=\left(M_{1}^{3 / 4} M_{2}^{3 / 4} M^{1 / 2} F^{1 / 4}\right)^{1 / 6}\left(M_{1} M_{2} M^{2 / 3}\right)^{5 / 6} M^{-1 / 18} .
\end{aligned}
$$

LEMMA 2. Let $a_{m}(m \sim M), b_{n}(n \sim N)$ be complex numbers of modulus $\leq 1$. We have

$$
\sum_{m \sim M} \sum_{\substack{n \sim N \\ v<m n \leq e v}} a_{m} b_{n}\left\{\psi\left(\frac{x+y}{m n}\right)-\psi\left(\frac{x}{m n}\right)\right\} \ll y x^{-5 \eta}
$$

for $1 / 2<\theta<2 / 3-\varepsilon$ and $v x^{-1 / 2+\varepsilon} \ll N \ll \max \left(x^{2-\varepsilon} v^{-3}, v^{1 / 7} x^{1 / 14-\varepsilon}\right)$. 
Proof. If the sum (2.1) is nonempty, then $M N \asymp v$. (This will frequently be used implicitly in the rest of the paper.)

In view of [1], Lemma 7, and the proof of [1], inequality (50), it suffices to establish

$$
T:=\sum_{h \sim H} \sum_{m \sim M} \sum_{n \sim N} a_{m} b_{n} e\left(\frac{h \zeta}{m n}\right) \ll v x^{-6 \eta}
$$

for $H \leq J, M N \asymp v, \zeta \asymp x$. (We have dispensed with the summation condition $v<m n \leq e v$; this is permissible just as in [1], Lemma 15.)

We now get the desired result by an appeal to Lemma 9 of [1] and Lemma 2 of [13].

Lemma 3. Let $(\kappa, \lambda)$ be an exponent pair. Let $0.64<\theta<0.72$. Then (2.1) holds for all $N$ satisfying

$$
v x^{-1 / 2+\varepsilon} \ll N \ll\left(x^{\lambda / 2-\kappa-1 / 4} v^{1+\kappa-\lambda}\right)^{1 /(1+\lambda-\kappa)} x^{-\varepsilon} .
$$

Proof. As in the previous lemma, it suffices to establish (2.2). According to Lemma 14 of [1], for $N$ satisfying (2.3) we have

$$
T^{2} \ll M^{2}(N H)^{1+\eta}+H^{1 / 2+\lambda} N^{3 / 2+\lambda-2 \kappa} M^{1 / 2-\kappa} x^{1 / 2+\kappa+2 \eta} .
$$

It is now easy to verify that (2.3) and (2.4) together imply (2.2). This completes the proof of Lemma 3.

We shall apply Lemma 3 with three exponent pairs:

(i) Let $\mu=89 / 560+\eta$. Then $(\mu, 1 / 2+\mu)$ is an exponent pair (Watt [19]). Let

$$
(\kappa, \lambda)=B A\left(\mu, \frac{1}{2}+\mu\right)=\left(\frac{1+2 \mu}{4+4 \mu}, \frac{1+2 \mu}{2+2 \mu}\right) .
$$

(ii) $(\kappa, \lambda)=B A^{3} B(0,1)=(11 / 30,8 / 15)$.

(iii) $(\kappa, \lambda)=B A^{4} B(0,1)=(13 / 31,16 / 31)$.

The corresponding expressions in (2.3) are respectively

$$
v^{(3+2 \mu) /(5+6 \mu)} x^{-(1+\mu) /(5+6 \mu)-\varepsilon}, \quad v^{5 / 7} x^{-3 / 10-\varepsilon}, \quad v^{14 / 17} x^{-3 / 8-\varepsilon} .
$$

Lemma 4. We have

$$
\sum_{h \sim H} \sum_{n \sim N} \sum_{\substack{M \leq m<M_{1} \\ v<m n \leq e v}} b_{n} e\left(\frac{h \zeta}{m n}\right) \ll v x^{-6 \eta}
$$

for

$$
\zeta \asymp x, \quad 1 / 2<\theta<3 / 4-\varepsilon, \quad M_{1} \leq 2 M, \quad\left|b_{n}\right| \leq 1, \quad H \leq J
$$

if either

$$
N<x^{1 / 3-\varepsilon}
$$


or

$$
x^{1 / 3-\varepsilon} \leq N \leq x^{3 / 8-\varepsilon} \text { and } H<v x^{\varepsilon-3 / 8} N^{-1 / 2} .
$$

Proof. This is a variant of an argument of Wu [20]. We apply Poisson's summation formula to the inner summation. As in the proof of [1], Lemma 4, the left-hand side of (2.5) is

$$
\frac{M v^{1 / 2}}{H^{1 / 2} x^{1 / 2}} \sum_{h \sim H} \sum_{n \sim N} \sum_{r} b_{n}^{\prime} c_{r} e\left(2\left(\frac{h r \zeta}{n}\right)^{1 / 2}\right)+O_{\varepsilon}\left(H N\left(\frac{M v^{1 / 2}}{H^{1 / 2} x^{1 / 2}}+L\right)\right),
$$

where $b_{n}^{\prime}, c_{r}$ have modulus $\ll 1$ and the summation range for $r$ is

$$
h \zeta n^{-1} \max \left(M_{1}^{-2},\left(\frac{e v}{n}\right)^{-2}\right)<r<h \zeta n^{-1} \min \left(M^{-2},\left(\frac{v}{n}\right)^{-2}\right) .
$$

We may readily verify that

$$
H N\left(\frac{M v^{1 / 2}}{H^{1 / 2} x^{1 / 2}}+L\right) \ll v x^{-6 \eta}
$$

As in the proof of Lemma 2, it now suffices to show that

$$
\sum_{h \sim H} \sum_{n \sim N} \sum_{r \sim R} b_{n}^{\prime \prime} c_{r}^{\prime} e\left(2\left(\frac{h r \zeta}{n}\right)^{1 / 2}\right) \ll M^{-1} v^{1 / 2} H^{1 / 2} x^{1 / 2-7 \eta},
$$

where

$$
R \asymp H x M^{-2} N^{-1}
$$

and $\left|b_{n}^{\prime \prime}\right| \leq 1,\left|c_{r}^{\prime}\right| \leq 1$. By a standard divisor argument, the sum (2.10) may be rewritten as

$$
\sum_{n \sim N} \sum_{k \asymp R H} b_{n}^{\prime \prime} a_{k} e\left(2\left(\frac{k \zeta}{n}\right)^{1 / 2}\right)
$$

where $a_{k} \ll x^{\eta}$.

We apply Lemma 1 with $M_{1} \asymp R H, M_{2}=1$ and $M$ replaced by $N$. It is easily verified that (2.10) is a consequence of (2.7) or (2.8). This completes the proof of Lemma 4 . The reader will note that (2.8) is unnecessarily strong. The reason for the form of the condition (2.8) will become clear when we prove Corollary 1 of Theorem 2 .

\section{Bilinear exponential sums}

LemMA 5. Let $M \leq N<N_{1} \leq M_{1}$. Let $a_{n}\left(M \leq n \leq M_{1}\right)$ be complex numbers. Then

$$
\left|\sum_{N<n \leq N_{1}} a_{n}\right| \leq \int_{-\infty}^{\infty} K(\phi)\left|\sum_{M<m \leq M_{1}} a_{m} e(\phi m)\right| d \phi
$$


with $K(\phi)=\min \left(M_{1}-M+1,(\pi|\phi|)^{-1},(\pi \phi)^{-2}\right)$ and

$$
\int_{-\infty}^{\infty} K(\phi) d \phi \leq 3 \log \left(2+M_{1}-M\right)
$$

Proof. This is Lemma 2.2 of Bombieri and Iwaniec [3].

Theorem 2. Let $\alpha, \beta$ be given nonzero real numbers, $\alpha \neq 1$. Let $X, Y$ $\geq 1$. Let $a_{x}(x \sim X)$ and $b_{y}(y \sim Y)$ be complex numbers of modulus $\leq 1$. Let

$$
S=\sum_{x \sim X} \sum_{y \sim Y} a_{x} b_{y} e\left(A x^{\alpha} y^{\beta}\right),
$$

where the positive number $F=A X^{\alpha} Y^{\beta}$ satisfies

$$
F<\min \left(Y^{2}, X Y^{1-3 \eta}\right) .
$$

Then for any natural number $Q, 1 \leq Q \leq Y^{1-\eta}$, we have

$$
\begin{aligned}
S \ll(X Y)^{3 \eta}\{ & X Y Q^{-1 / 2}+X Y^{3 / 2} F^{-1 / 2} Q^{-1 / 2}+F^{-1 / 6} Q^{-1 / 3} X Y^{13 / 12} \\
& +F^{-3 / 8} Q^{-5 / 16} X Y^{11 / 8}+F^{1 / 4} Q^{1 / 8} X^{1 / 2} Y^{3 / 4} \\
& \left.+F^{1 / 3} Q^{1 / 6} X^{1 / 2} Y^{7 / 12}+F^{1 / 8} Q^{3 / 16} X^{1 / 2} Y^{7 / 8}\right\} .
\end{aligned}
$$

Proof. We may suppose that $Y>c_{0}(\eta)$. We begin in the same way as Liu [13], proof of Lemma 4. By Cauchy's inequality and a "Weyl shift" (see e.g. Graham and Kolesnik [10], Lemma 2.5),

$$
\begin{aligned}
|S|^{2} & \leq X \sum_{x \sim X}\left|\sum_{y \sim Y} b_{y} e\left(A x^{\alpha} y^{\beta}\right)\right|^{2} \\
& \ll \frac{(X Y)^{2}}{Q}+\frac{X Y}{Q} \sum_{0<|q| \leq Q} \sum_{y, y+q \sim Y} \bar{b}_{y} b_{y+q} \sum_{x \sim X} e\left(A x^{\alpha} t(y, q)\right) .
\end{aligned}
$$

Here $t(y, q)=(y+q)^{\beta}-y^{\beta}$. After splitting the range of $q$ into dyadic intervals, we obtain

$$
Y^{-\eta}|S|^{2} \ll \frac{(X Y)^{2}}{Q}+\frac{X Y}{Q}\left|\sum_{q \sim Q_{1}} \sum_{y, y+q \sim Y} \bar{b}_{y} b_{y+q} \sum_{x \sim X} e\left(A x^{\alpha} t(y, q)\right)\right|
$$

for some $Q_{1}, 1 \leq Q_{1} \leq Q$.

There are now two cases to consider.

$$
\begin{aligned}
\text { Case } 1: Q_{1} & \leq Y^{2 \eta} \text {. Now } \\
& \frac{d}{d x}\left(A x^{\alpha} t(y, q)\right) \ll F Q_{1} Y^{-1} X^{-1} \ll Y^{-\eta}
\end{aligned}
$$

by (3.1). Lemmata 4.2 and 4.8 of [17] yield

$$
\sum_{x \sim X} e\left(A x^{\alpha} t(y, q)\right) \ll\left(F Q_{1}\right)^{-1} X Y
$$


and

$$
\begin{aligned}
Y^{-\eta}|S|^{2} & \ll \frac{(X Y)^{2}}{Q}+\frac{X Y^{2}}{Q} Q_{1}\left(F Q_{1}\right)^{-1} X Y \\
& \ll(X Y)^{2}\left(Q^{-1}+Y F^{-1} Q^{-1}\right),
\end{aligned}
$$

so that the theorem is true in this case.

Cas e 2: $Q_{1}>Y^{2 \eta}$. We apply a sharp form of the Poisson summation formula to the innermost sum (Min [14], Theorem 2.2):

$$
\begin{aligned}
\sum_{x \sim X} e\left(A x^{\alpha} t\right)= & \sum_{u \in I} C_{1}\left|(A t)^{\gamma} u^{-1 / 2-\gamma}\right| e\left(C_{2}(A t)^{2 \gamma} u^{1-2 \gamma}\right) \\
& +O\left(\min \left(\left(\frac{X^{2} Y}{Q_{1} F}\right)^{1 / 2}, \frac{1}{\left\|g_{1}(y, q)\right\|}+\frac{1}{\left\|g_{2}(y, q)\right\|}\right)\right) \\
& +\frac{X Y}{Q_{1} F}+(X Y)^{\eta} .
\end{aligned}
$$

Here $I=\left[C_{3} A X^{\alpha-1}|t|, C_{4} A X^{\alpha-1}|t|\right], \gamma=1 /(2(1-\alpha)), 2 \gamma(2 \gamma-1) \neq 0$, $g_{1}=\alpha A X^{\alpha-1} t$ and $g_{2}=\alpha A(2 X)^{\alpha-1} t$. (The constants $C_{1}, C_{2}, \ldots$ depend only on $\alpha$.)

For fixed $q \sim Q_{1}$, we have

$$
\begin{aligned}
& \sum_{j=1}^{2} \sum_{y \sim Y} \min \left(\left(X^{2} Y Q_{1}^{-1} F^{-1}\right)^{1 / 2}, \frac{1}{\left\|g_{j}(y, q)\right\|}\right) \\
& \quad \ll\left(1+F Q_{1} X^{-1} Y^{-1}\right)\left(\left(X^{2} Y Q_{1}^{-1} F^{-1}\right)^{1 / 2}+F^{-1} Q_{1}^{-1} X Y^{2+\eta}\right)
\end{aligned}
$$

by a variant of Lemma 9 of Vinogradov [18], Chapter I. Thus if we sum (3.2) over $y$, interchange summations, and apply a partial summation, we find that

$$
\begin{aligned}
& (X Y)^{-2 \eta}|S|^{2} \\
\ll & (X Y)^{2} Q^{-1}+X^{2} Y^{3 / 2} Q^{-1 / 2} F^{-1 / 2}+F^{-1} Q^{-1} X^{2} Y^{3} \\
& +F^{1 / 2} Q^{1 / 2} X Y^{1 / 2}+X Y^{2} \\
& +X Y Q^{-1}\left(1+F Q_{1} X^{-1} Y^{-1}\right)\left(A Q_{1} Y^{\beta-1}\right)^{\gamma}\left(X Y F^{-1} Q_{1}^{-1}\right)^{\gamma+1 / 2}\left|S_{1}\right| .
\end{aligned}
$$

Here, for some fixed $u \asymp F Q_{1} X^{-1} Y^{-1}$,

$$
S_{1}=\sum_{y} \sum_{q \sim Q_{1}} \bar{b}_{y} b_{y+q} e\left(C_{2}(A t)^{2 \gamma} u^{1-2 \gamma}\right),
$$

where the outer summation is taken over a subinterval of $(Y, 2 Y]$.

By Lemma 5, we have

$$
(X Y)^{-\eta}\left|S_{1}\right| \ll \sum_{y \sim Y}\left|\sum_{q \sim Q_{1}} b_{y+q} e(\phi q) e\left(C_{2}(A t)^{2 \gamma} u^{1-2 \gamma}\right)\right|
$$


for some real number $\phi$ independent of $y$ and $q$. Applying Cauchy's inequality and a Weyl shift, we have

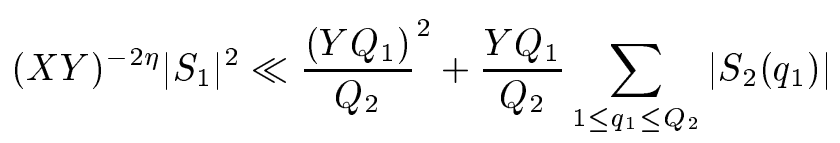

with

$$
S_{2}\left(q_{1}\right)=\sum_{y \sim Y} \sum_{q, q+q_{1} \sim Q_{1}} b_{y+q} b_{y+q+q_{1}} e\left(t_{1}\right) .
$$

Here

$$
t_{1}=t_{1}\left(y, q, q_{1}\right)=C_{2} u^{1-2 \gamma} A^{2 \gamma}\left(t\left(y, q+q_{1}\right)^{2 \gamma}-t(y, q)^{2 \gamma}\right) .
$$

The choice of $Q_{2}\left(1 \leq Q_{2} \leq Q_{1}\right)$ is at our disposal. For reasons which should shortly become clear, we take

$$
Q_{2}=\min \left(Q_{1}^{(1-\eta) / 2},\left(Q_{1} Y^{2-\eta} F^{-1}\right)^{1 / 3}\right) .
$$

We now estimate $S_{2}$ for a given value of $q_{1}$. (We suppress dependence on $q_{1}$.) Writing $y+q=z$, we get

$$
S_{2}=\sum_{q, q+q_{1} \sim Q_{1}} \sum_{z-q \sim Y} \bar{b}_{z} b_{z+q_{1}} e(T)
$$

with

$$
T=T(z, q)=C_{2} u^{1-2 \gamma} A^{2 \gamma}\left(t\left(z-q, q+q_{1}\right)^{2 \gamma}-t(z-q, q)^{2 \gamma}\right) .
$$

Applying Lemma 5 once more gives

$$
(X Y)^{-\eta}\left|S_{2}\right| \ll \sum_{Y / 2<z<2 Y}\left|\sum_{q \sim Q_{1}} e(T(z, q))\right| .
$$

A final application of Cauchy's inequality and a Weyl shift yields

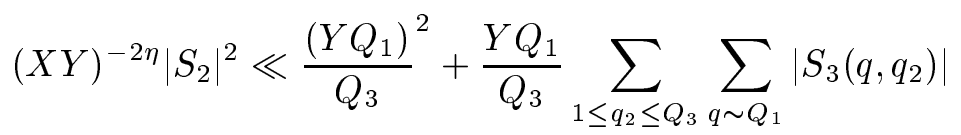

with $Q_{3}=Q_{2}^{2} \leq Q_{1}^{1-\eta}$ and

$$
S_{3}\left(q, q_{2}\right)=\sum_{Y / 2<z<2 Y} e\left(T(z, q)-T\left(z, q+q_{2}\right)\right) .
$$

Now it is easy to verify that, in the last sum,

$$
\frac{d}{d z}\left(T(z, q)-T\left(z, q+q_{2}\right)\right) \asymp F q_{1} q_{2} Q_{1}^{-1} Y^{-2} \ll F Q_{2}^{3} Q_{1}^{-1} Y^{-2} \ll Y^{-\eta} .
$$

By Lemmata 4.2 and 4.8 of [17], we have

$$
S_{3}\left(q, q_{2}\right) \ll\left(F q_{1} q_{2} Q_{1}^{-1} Y^{-2}\right)^{-1} .
$$


With (3.6), (3.5), this yields

$$
\begin{aligned}
& (X Y)^{-3 \eta}\left|S_{2}\right|^{2} \ll \frac{Y^{2} Q_{1}^{2}}{Q_{2}^{2}}+\frac{Y^{3} Q_{1}^{3}}{Q_{2}^{2} q_{1} F}, \\
& (X Y)^{-4 \eta}\left|S_{1}\right|^{2} \ll \frac{Y^{2} Q_{1}^{2}}{Q_{2}}+\frac{Y^{5 / 2} Q_{1}^{5 / 2}}{Q_{2}^{3 / 2} F^{1 / 2}} .
\end{aligned}
$$

Using the definition of $Q_{2}$, we get

$$
\begin{aligned}
(X Y)^{-5 \eta}\left|S_{1}\right|^{2} \ll & Y^{2} Q_{1}^{3 / 2}+Y^{4 / 3} Q_{1}^{5 / 3} F^{1 / 3} \\
& +Y^{5 / 2} Q_{1}^{7 / 4} F^{-1 / 2}+Y^{3 / 2} Q_{1}^{2} .
\end{aligned}
$$

Since $Q_{1}<Y$, the last term may be omitted. Recalling (3.4), and noting that

we obtain

$$
\left(A Q_{1} Y^{\beta-1}\right)^{\gamma}\left(X Y F^{-1} Q_{1}^{-1}\right)^{\gamma}=X^{1 / 2},
$$

$$
\begin{aligned}
(X Y)^{-5 \eta}|S|^{2} \ll & (X Y)^{2} Q^{-1}+X^{2} Y^{3 / 2} Q^{-1 / 2} F^{-1 / 2} \\
& +F^{-1} Q^{-1} X^{2} Y^{3}+F^{1 / 2} Q^{1 / 2} X Y^{1 / 2}+X Y^{2} \\
& +X^{2} Y^{3 / 2} Q^{-1} F^{-1 / 2} Q_{1}^{-1 / 2}\left(1+F Q_{1} X^{-1} Y^{-1}\right) \\
& \times\left(Y Q_{1}^{3 / 4}+Y^{2 / 3} Q_{1}^{5 / 6} F^{1 / 6}+Y^{5 / 4} Q_{1}^{7 / 8} F^{-1 / 4}\right) \\
\ll & X^{2} Y^{2} Q^{-1}+X^{2} Y^{3 / 2} Q^{-1 / 2} F^{-1 / 2}+F^{-1} Q^{-1} X^{2} Y^{3} \\
& +F^{1 / 2} Q^{1 / 2} X Y^{1 / 2}+X Y^{2}+X^{2} Y^{5 / 2} Q^{-3 / 4} F^{-1 / 2} \\
& +F^{-1 / 3} Q^{-2 / 3} X^{2} Y^{13 / 6}+F^{-3 / 4} Q^{-5 / 8} X^{2} Y^{11 / 4} \\
& +X Y^{3 / 2} Q^{1 / 4} F^{1 / 2}+X Y^{7 / 6} F^{2 / 3} Q^{1 / 3}+X Y^{7 / 4} F^{1 / 4} Q^{3 / 8} \\
= & T_{1}+\ldots+T_{11}, \text { say. }
\end{aligned}
$$

Clearly $T_{4} \leq T_{9}$ and $T_{2} \leq T_{6}=T_{1}^{1 / 3} T_{8}^{2 / 3} \leq \max \left(T_{1}, T_{8}\right)$. We may suppose that $T_{3}<X^{2} Y^{2}$; consequently, $Y<F Q$ and $T_{5}<T_{11}$. The result follows in Case 2. This completes the proof of Theorem 2.

Corollary 1. We have, for $1 / 2<\theta \leq 3 / 4-\varepsilon$,

$$
\sum_{M \leq m<M_{1}} \sum_{\substack{n \sim N \\ v<m n \leq e v}} b_{n}\left\{\psi\left(\frac{x+y}{m n}\right)-\psi\left(\frac{x}{m n}\right)\right\} \ll y x^{-5 \eta}
$$

whenever $\left|b_{n}\right| \leq 1, M_{1} \leq 2 M$ and

$$
N \leq x^{3 / 8-\varepsilon} .
$$

Proof. As in the proof of Lemma 2, it suffices to show that (2.5) holds when $\zeta \asymp x, H \leq J$. In view of Lemma 4 , we may suppose that

$$
x^{1 / 3-\varepsilon} \leq N \leq x^{3 / 8-\varepsilon}
$$


and that

$$
H \geq v x^{\varepsilon-3 / 8} N^{-1 / 2} .
$$

As in the proof of Lemma 4, we only have to prove that the left-hand side of $(2.12)$ is

$$
\ll M^{-1} v^{1 / 2} H^{1 / 2} x^{1 / 2-6 \eta} .
$$

We apply Theorem 2 with

$$
X=N, \quad Y \asymp H^{2} x v^{-2} N, \quad F \asymp H x v^{-1}, \quad Q=\left[x^{1 / 4}\right] .
$$

It is easy to deduce from (3.10) that $Q<Y^{1-\eta}$ and that (3.1) holds. The bound (3.11) may readily be verified, and Corollary 1 is proved.

Corollary 2. The formula (1.6) holds.

Proof. An examination of the proof of [1], Proposition 1, reveals that it suffices to prove that (3.7) holds whenever $\left|b_{n}\right| \leq 1, M_{1} \leq 2 M$ and

$$
M>x^{1 / 4-6 \eta}, \quad v<x^{3 / 5-\varepsilon} .
$$

Now (3.12) implies $N \ll v M^{-1} \ll x^{7 / 20+2 \varepsilon}$. Hence Corollary 2 follows from Corollary 1.

4. The Rosser-Iwaniec sieve. Let $\tau(\theta)$ be defined to be $2-3 \theta$ in $(3 / 5-\varepsilon, 27 / 44],(2 \theta+1) / 14$ in $(27 / 44,0.642],(3+2 \mu) \theta /(5+6 \mu)-(1+\mu) /(5+$ $6 \mu)$ in $(0.642,0.671], 5 \theta / 7-3 / 10$ in $(0.671,357 / 520]$ and $14 \theta / 17-3 / 8$ in $(357 / 520,0.7]$. Let

$$
a=x^{\theta-1 / 2+\varepsilon}, \quad b=x^{\tau(\theta)-\varepsilon}, \quad b a^{-1}=x^{g}, \quad I=[a, b] .
$$

Then

$$
\text { The bound (2.1) holds for } N \in I \text {. }
$$

This is a consequence of Lemma 2 for $\theta \leq 0.642$. We use the remarks following Lemma 3 for the remaining intervals. When $\theta \geq 0.661$, we shall apply the Rosser-Iwaniec sieve as in [1] to bound $S(\mathcal{A}, z)$ from above. Here $z=D^{1 / 3}$, the "level of distribution" $D$ being defined as follows. For $0.661<$ $\theta \leq 0.7$, let

$$
D_{0}=x^{-5 \varepsilon} \min \left(b^{3} a^{-1}, x^{3 / 4} a^{-2}\right)
$$

and

$$
D=\max \left(D_{0}, x^{3 / 8+g-5 \varepsilon}\right)=x^{\varrho(\theta)}, \quad \text { say. }
$$

For $0.7<\theta \leq 0.732$, let $D=x^{\varrho(\theta)}$, where $\varrho(\theta)=3 / 8-4 \varepsilon$.

The interval of $\theta$ in which $D=D_{0}$ is rather short, $0.6825<\theta<$ $0.6854 \ldots$, but the device seems worth including, partly because analogous situations may occur in other sieve problems. 
Lemma 6. Let $A_{1}, \ldots, A_{t}$ be positive numbers with $A_{1} \gg \ldots \gg A_{t} \geq 1$,

$$
A_{1} \ldots A_{2 j} A_{2 j+1}^{3} \leq D^{1+\eta} \quad(0 \leq j \leq(t-1) / 2) .
$$

Then for $0.661<\theta \leq 0.7$, either

$$
A_{1} \ldots A_{t}<x^{3 / 8-2 \varepsilon}
$$

or some set $\mathcal{S} \subset\{1, \ldots, t\}$ satisfies

$$
\prod_{i \in \mathcal{S}} A_{i} \in I
$$

For $0.7<\theta \leq 0.73$, the inequality (4.3) holds.

Proof. The lemma is obvious for $\theta>0.7$, since (4.2) implies that $A_{1} \ldots A_{t} \ll D^{1+\eta} \ll x^{3 / 8-3 \varepsilon}$. Now let $\theta \in(0.661,0.7]$. Suppose that neither (4.3) nor (4.4) holds; we shall obtain a contradiction.

Suppose first that $D=x^{3 / 8+g-5 \varepsilon}$. By Lemma 5 of Fouvry [6], with $Y_{i}=A_{i}, W=D^{1+2 \eta}, U=a, V=b$, we have

$$
A_{1} \ldots A_{t} \leq D^{1+2 \eta} a b^{-1}<x^{3 / 8-2 \varepsilon} \text {. }
$$

This is absurd.

Next, suppose that $D=D_{0}$. As in [1], p. 215, we partition $A_{1} \ldots A_{t}$ into two products $P$ and $Q$, with

$$
P \leq D_{0}^{1 / 2+\eta}, \quad Q \leq D_{0}^{1 / 2+\eta} .
$$

Suppose if possible that $P \leq b$. Then $P<a$. Now

$$
A_{1} \ldots A_{t}=P Q<a D_{0}^{1 / 2+\eta}<x^{3 / 8-2 \varepsilon} .
$$

This is absurd, so $P>b$. Similarly, $Q>b$.

Let $P^{\prime}$ be the subproduct of $P$ formed from those $A_{i}$ that exceed $b a^{-1}$; define $Q^{\prime}$ similarly. Since (4.4) never holds, it is clear that $P^{\prime} \geq b$ and $Q^{\prime} \geq b$. If $P^{\prime} Q^{\prime}=A_{j_{1}} \ldots A_{j_{r}}$ with $j_{1}>\ldots>j_{r}$ then, from (4.2),

$$
D^{1+\eta} \geq P^{\prime} Q^{\prime} A_{j_{r}} \geq b^{2} \cdot b a^{-1},
$$

which is absurd. This completes the proof of Lemma 6 .

Lemma 7. Let $z=D^{1 / 3}$. Then

$$
S(\theta) \leq S(\mathcal{A}, z) \lesssim \frac{2}{\varrho(\theta)} \cdot \frac{y}{L}
$$

P r o of. In view of Lemma 6 and (4.1), this follows in exactly the same way as [1], Lemma 16. (The condition (63) of [1] is obviously satisfied.)

5. An alternative sieve. In this section and the next we suppose that

$$
3 / 5-\varepsilon<\theta \leq 0.661 \text {. }
$$


Let

$$
\mathcal{B}=\{n: v<n \leq e v\}, \quad \omega(n)=y / n \quad(n \in \mathcal{B}) .
$$

For $\mathcal{E}=\mathcal{A}$ or $\mathcal{B}$, let $\mathcal{E}_{m}=\{n: m n \in \mathcal{E}\}$. We write

$$
S\left(\mathcal{B}_{m}, u\right)=\sum_{\substack{m n \in \mathcal{B} \\ Q(n) \geq u}} \omega(n m)
$$

whenever $Q(m) \geq u$. The quantity $S(\mathcal{B}, u)$ will act as a model for $S(\mathcal{A}, u)$.

We let $w(u)$ be Buchstab's function, so that

$$
w(u)=1 / u \quad(1 \leq u \leq 2), \quad(u w(u))^{\prime}=w(u-1) \quad(u>2) .
$$

As $u \rightarrow \infty$, we have

$$
w(u)=e^{-\gamma}+O(G(1 / u)) .
$$

See Cheer and Goldston [4].

Lemma 8. For $m \leq v^{1 / 2}$ and $x^{\varepsilon} \leq \lambda \leq v / m, Q(m) \geq \lambda$, we have

$$
S\left(\mathcal{B}_{m}, \lambda\right)=w\left(\frac{\log (v / m)}{\log \lambda}\right) \frac{y}{m \log \lambda}\left(1+O_{\varepsilon}\left(L^{-1}\right)\right) .
$$

Proof. Let $\Gamma(u)=|\{n \leq u: Q(n) \geq \lambda\}|$. For $u \in[\gamma / m, e v / m]$, we have

$$
\Gamma(u)=w\left(\frac{\log (v / m)}{\log \lambda}\right) \frac{u}{\log \lambda}\left(1+O_{\varepsilon}\left(L^{-1}\right)\right)
$$

(see Friedlander [8]). Consequently,

$$
\begin{aligned}
S\left(\mathcal{B}_{m}, \lambda\right) & =\frac{y}{m} \sum_{\substack{m n \in \mathcal{B} \\
Q(n) \geq \lambda}} \frac{1}{n}=\frac{y}{m} \int_{v / m}^{e v / m} \frac{d \Gamma(u)}{u} \\
& =\frac{y}{m}\left\{\left[\frac{\Gamma(u)}{u}\right]_{v / m}^{e v / m}+\int_{v / m}^{e v / m} \frac{\Gamma(u)}{u^{2}} d u\right\} \\
& =w\left(\frac{\log (v / m)}{\log \lambda}\right) \frac{y}{m \log \lambda}\left(1+O_{\varepsilon}\left(L^{-1}\right)\right) .
\end{aligned}
$$

This completes the proof of Lemma 8 .

Lemma 9. For $M \leq x^{3 / 8-\varepsilon}$, we have

$$
\sum_{m \leq M} a_{m}\left|\mathcal{A}_{m}\right|=y \sum_{m \leq M} \frac{a_{m}}{m}+O_{\varepsilon}\left(y x^{-4 \eta}\right)
$$

whenever $\left|a_{m}\right| \leq 1$. 
Proof. The left-hand side of (5.2) is

$$
\begin{aligned}
\sum_{m \leq M} a_{m} \sum_{v<m n \leq e v} & \sum_{\substack{k \\
x<m n k \leq x+y}} 1 \\
= & \sum_{m \leq M} a_{m} \sum_{\substack{n \\
v<m n \leq e v}}\left(\frac{y}{m n}-\psi\left(\frac{x+y}{m n}\right)+\psi\left(\frac{x}{m n}\right)\right) \\
= & y \sum_{m \leq M} \frac{a_{m}}{m} \sum_{\substack{n \\
v<m n \leq e v}} \frac{1}{n}+O_{\varepsilon}\left(y x^{-4 \eta}\right)
\end{aligned}
$$

by Corollary 1 of Theorem 2. We may now easily complete the proof (compare [1], p. 210).

LEMMA 10. Let $M \leq x^{3 / 8-2 \varepsilon}, 0 \leq a_{m} \leq 1, a_{m}=0$ unless $Q(m) \geq x^{\eta}$ $(m=1, \ldots, M)$. Then

$$
\sum_{m \leq M} a_{m} S\left(\mathcal{A}_{m}, x^{\eta}\right)=\sum_{m \leq M} a_{m} S\left(\mathcal{B}_{m}, x^{\eta}\right)\left(1+O\left(G\left(\frac{\varepsilon}{\eta}\right)\right)\right)+O_{\varepsilon}\left(y x^{-3 \eta}\right) .
$$

P r o of. We apply Lemma 8 of Baker, Harman and Rivat [2], with $z, y$ replaced by $x^{\eta}, x^{\varepsilon}$, taking $\mathcal{E}=\mathcal{A}_{m}$ and $Y=y / m$. In the notation of [2], we have $\sigma=\varepsilon$, hence $\sigma^{50} \log \left(x^{\eta}\right)>1$. We deduce that, whenever $a_{m} \neq 0$,

$$
S\left(\mathcal{A}_{m}, x^{\eta}\right)=\frac{y}{m} V\left(x^{\eta}\right)\left(1+O\left(G\left(\frac{\varepsilon}{\eta}\right)\right)\right)+O\left(\sum_{\substack{d<x^{\varepsilon} \\ p \mid d \Rightarrow p<x^{\eta}}}|| \mathcal{A}_{m d}\left|-\frac{y}{m d}\right|\right) .
$$

(Note that $\mathcal{E}_{d}=\mathcal{A}_{m d}$ because $(m, d)=1$ here.)

By a divisor argument (compare [2]) there are numbers $c_{j} \ll x^{\eta}$ for which

$$
\begin{aligned}
& \sum_{m \leq M} a_{m} S\left(\mathcal{A}_{m}, x^{\eta}\right) \\
& \quad=y V\left(x^{\eta}\right) \sum_{m \leq M} \frac{a_{m}}{m}\left(1+O\left(G\left(\frac{\varepsilon}{\eta}\right)\right)\right)+O\left(\sum_{j<x^{3 / 8-\varepsilon}} c_{j}\left(\left|\mathcal{A}_{j}\right|-\frac{y}{j}\right)\right) \\
& \quad=y V\left(x^{\eta}\right) \sum_{m \leq M} \frac{a_{m}}{m}\left(1+O\left(G\left(\frac{\varepsilon}{\eta}\right)\right)\right)+O_{\varepsilon}\left(y x^{-3 \eta}\right)
\end{aligned}
$$

by Lemma 9 .

Combining Lemma 8 with (5.1), we have

$$
S\left(\mathcal{B}_{m}, x^{\eta}\right)=\frac{y}{m \eta L}\left\{e^{-\gamma}+O\left(G\left(\frac{\varepsilon}{\eta}\right)\right)\right\} .
$$


In view of the approximation

$$
V\left(x^{\eta}\right)=\frac{e^{-\gamma}}{\eta L}\left\{1+O_{\varepsilon}\left(L^{-1}\right)\right\},
$$

we obtain the desired result.

Let $I$ be the interval defined at the beginning of Section 4 .

Lemma 11. For $N \in I,\left|a_{m}\right| \leq 1,\left|b_{n}\right| \leq 1$, we have

$$
\sum_{\substack{m n \in \mathcal{A} \\ m \sim M, n \sim N}} a_{m} b_{n}=y \sum_{\substack{m n \in \mathcal{B} \\ m \sim M, n \sim N}} \frac{a_{m} b_{n}}{m n}+O_{\varepsilon}\left(y x^{-5 \eta}\right) .
$$

Proof. As in Lemma 9, the left side is

$$
\begin{aligned}
\sum_{\substack{m n \in \mathcal{B} \\
m \sim M, n \sim N}} a_{m} b_{n}\left(\frac{y}{m n}-\psi\left(\frac{x+y}{m n}\right)\right. & \left.+\psi\left(\frac{x}{m n}\right)\right) \\
& =y \sum_{\substack{m n \in \mathcal{B} \\
m \sim M, n \sim N}} \frac{a_{m} b_{n}}{m n}+O_{\varepsilon}\left(y x^{-5 \eta}\right)
\end{aligned}
$$

from (4.1). This establishes Lemma 11.

Lemma 12. Let $h \geq 1$ be given and suppose that $\mathcal{D} \subset\{1, \ldots, h\}$ and $M \in I, M_{1}<2 M$. Then

$$
\begin{aligned}
\sum_{p_{1}} \ldots \sum_{p_{h}}^{*} S\left(\mathcal{A}_{p_{1} \ldots p_{h}},\right. & \left.p_{1}\right) \\
& =\sum_{p_{1}} \ldots \sum_{p_{h}}^{*} S\left(\mathcal{B}_{p_{1} \ldots p_{h}}, p_{1}\right)+O_{\varepsilon}\left(y x^{-4 \eta}\right) .
\end{aligned}
$$

Here ${ }^{*}$ indicates that $p_{1}, \ldots, p_{h}$ satisfy

$$
\begin{gathered}
x^{\eta} \leq p_{1}<\ldots<p_{h}, \\
M \leq \prod_{j \in \mathcal{D}} p_{j}<M_{1}
\end{gathered}
$$

together with no more than $\varepsilon^{-1}$ further conditions of the form

$$
R \leq \prod_{j \in \mathcal{F}} p_{j} \leq S
$$

Proof. The left-hand side of (5.3) is

$$
\sum_{p_{1}} \ldots \sum_{p_{h}}^{*} \sum_{j=1}^{t} \sum_{q_{1}} \ldots \sum_{q_{j}} 1
$$


where $t<\eta^{-1}$, and the inner summation extends over primes $q_{1}, \ldots, q_{j}$ satisfying

$$
p_{1} \leq q_{1} \leq \ldots \leq q_{j}, \quad p_{1} \ldots p_{h} q_{1} \ldots q_{j} \in \mathcal{A} .
$$

This would be the type of sum estimated in Lemma 11, if we could disentangle the interactions between the variables $p_{1}, \ldots, p_{h}, q_{1}, \ldots, q_{j}$. The procedure for doing this via the truncated Perron formula in [1] (proof of Lemma 11) may be applied here. Accordingly the left-hand side of (5.3) is

$$
\sum_{p_{1}} \ldots \sum_{p_{h}}^{*} \sum_{j=1}^{t} \sum_{q_{1}} \ldots \sum_{q_{j}} \frac{y}{p_{1} \ldots p_{h} q_{1} \ldots q_{j}}+O_{\varepsilon}\left(y x^{-4 \eta}\right) .
$$

Naturally we may also obtain the formula (5.7) for the sum

$$
\sum_{p_{1}} \ldots \sum_{p_{h}}^{*} S\left(\mathcal{B}_{p_{1} \ldots p_{h}}, p_{1}\right)
$$

and Lemma 12 follows.

LEMMA 13. Let $M \leq a / 2$ and $N \leq x^{3 / 8-2 \varepsilon} /(2 a)$. Let $M \leq M_{1} \leq 2 M$ and $N \leq N_{1} \leq 2 N$. Let $x^{\eta}<z \leq b a^{-1}$. Suppose that $\{1, \ldots, h\}$ partitions into two sets $\mathcal{C}$ and $\mathcal{D}$. Then

$$
\sum_{p_{1}} \ldots \sum_{p_{h}}^{*} S\left(\mathcal{A}_{p_{1} \ldots p_{h}}, z\right)=\sum_{p_{1}} \ldots \sum_{p_{h}}^{*} S\left(\mathcal{B}_{p_{1} \ldots p_{h}}, z\right)(1+O(\varepsilon)) .
$$

Here $^{*}$ indicates that $p_{1}, \ldots, p_{h}$ satisfy

$$
\begin{gathered}
z \leq p_{1}<\ldots<p_{h}, \\
M \leq \prod_{j \in \mathcal{C}} p_{j}<M_{1}, \quad N \leq \prod_{j \in \mathcal{D}} p_{j}<N_{1}
\end{gathered}
$$

together with no more than $\varepsilon^{-1}$ further conditions of the form (5.6).

We remark that the case $h=0, \mathcal{C}$ and $\mathcal{D}$ empty is permitted.

Proof. Let us write $\mathbf{p}=\left(p_{1}, \ldots, p_{h}\right)$ and $m=\prod_{j \in \mathcal{C}} p_{j}, n=\prod_{j \in \mathcal{D}} p_{j}$. Buchstab's identity

$$
S\left(\mathcal{E}, z_{1}\right)=S\left(\mathcal{E}, z_{2}\right)-\sum_{z_{2} \leq p<z_{1}} S\left(\mathcal{E}_{p}, p\right)
$$

applies to both $\mathcal{A}_{m n}$ and $\mathcal{B}_{m n}\left(2 \leq z_{2}<z_{1}\right)$. In particular,

$$
\sum_{\mathbf{p}}^{*} S\left(\mathcal{A}_{m n}, z\right)=\sum_{\mathbf{p}}^{*} S\left(\mathcal{A}_{m n}, x^{\eta}\right)-\sum_{\mathbf{p}}^{*} \sum_{x^{\eta} \leq q_{1}<z} S\left(\mathcal{A}_{m n q_{1}}, q_{1}\right) .
$$

The first term on the right has an asymptotic formula by Lemma 10 .

The subsum of the other term on the right for which $m q_{1} \geq a$ has an asymptotic formula by Lemma 12 , since $m q_{1} \leq 2 M z \leq b$. 
To the residual sum in which $m q_{1}<a$, we apply Buchstab once more. If we continue in this fashion, the $j$ th step is the identity

$$
\begin{aligned}
\sum_{j} & :=\sum_{\mathbf{p}}^{*} \sum_{(5.11)} S\left(\mathcal{A}_{m n q_{1} \ldots q_{j}}, q_{j}\right) \\
& =\sum_{\mathbf{p}}^{*} \sum_{(5.11)} S\left(\mathcal{A}_{m n q_{1} \ldots q_{j}}, x^{\eta}\right)-\sum_{\mathbf{p}}^{*} \sum_{(5.12)} S\left(\mathcal{A}_{m n q_{1} \ldots q_{j+1}}, q_{j+1}\right)
\end{aligned}
$$

with summation conditions

$$
\begin{gathered}
x^{\eta} \leq q_{j}<\ldots<q_{1}<z, \quad m q_{1} \ldots q_{j}<a, \\
x^{\eta} \leq q_{j+1}<q_{j}<\ldots<q_{1}<z, \quad m q_{1} \ldots q_{j}<a .
\end{gathered}
$$

The first sum on the right has an asymptotic formula by Lemma 10, since the variables satisfy

$$
m n q_{1} \ldots q_{j}<a\left(x^{3 / 8-2 \varepsilon} / a\right)=x^{3 / 8-2 \varepsilon} .
$$

The subsum of the second sum on the right, given by $m q_{1} \ldots q_{j+1} \geq a$, has an asymptotic formula by Lemma 12, since

$$
m q_{1} \ldots q_{j+1}<a q_{j+1}<a z \leq b .
$$

The residual sum is $\sum_{j+1}$. After $O\left(\eta^{-1}\right)$ steps the residual sum is clearly empty, giving a decomposition of $\sum_{\mathrm{p}}^{*} S\left(\mathcal{A}_{m n}, z\right)$ into a main term and an error term, say $E$.

A corresponding decomposition with the error term applies to $\sum_{\mathrm{p}}^{*} S\left(\mathcal{B}_{m n}, z\right)$, and to complete the proof we must show that $E$ is of acceptable size. Just as in [2], proof of Lemma 12, we have

$$
E=O\left(\eta^{-1} 2^{1 / \eta} G\left(\frac{\varepsilon}{\eta}\right) \sum_{\mathbf{p}}^{*} S\left(\mathcal{A}_{m n}, z\right)\right)=O\left(\varepsilon \sum_{\mathbf{p}}^{*} S\left(\mathcal{A}_{m n}, z\right)\right)
$$

since

$$
\eta^{-1} 2^{1 / \eta} G\left(\frac{\varepsilon}{\eta}\right)<\exp \left(\frac{1}{\eta}-\frac{\varepsilon}{\eta} \log \left(\frac{\varepsilon}{\eta}\right)\right)<\exp \left(\frac{-1}{\eta}\right) .
$$

This completes the proof of Lemma 13.

Lemma 14. Let $x^{3 \varepsilon} P<\max \left(b^{2} / a, x^{7 / 8-\theta}\right)$. Then

$$
\sum_{p \sim P} S\left(\mathcal{A}_{p}, b a^{-1}\right)=\sum_{p \sim P} S\left(\mathcal{B}_{p}, b a^{-1}\right)(1+O(\varepsilon)) .
$$

Remark. $b^{2} / a>x^{7 / 8-\theta}$ for $\theta<29 / 48=0.60416 \ldots$

Proof of Lemma 14. In view of Lemma 13 we need only concern ourselves with the case

$$
x^{7 / 8-\theta}<x^{3 \varepsilon} P<b^{2} / a .
$$


We can begin the proof as in Lemma 13, but we cannot continue when we reach a point where

$$
P q_{1} \ldots q_{n}>x^{3 / 8-2 \varepsilon}, \quad q_{1} \ldots q_{n}<a .
$$

At such a stage we note that

$$
\sum_{\substack{q_{j} \sim Q_{j} \\ p \sim P}} S\left(\mathcal{A}_{p q_{1} \ldots q_{n}}, q_{n}\right)=\sum_{\substack{q_{j} \sim Q_{j} \\ r}} S\left(\mathcal{A}_{r q_{1} \ldots q_{n}},(2 P)^{1 / 2}\right),
$$

where $r$ has no prime factors below $q_{n}$, and $v /(2 P) \leq r q_{1} \ldots q_{n}<e v / P$. In view of (5.14) we thus obtain

$$
r \ll v x^{2 \varepsilon-3 / 8}=x^{\theta+2 \varepsilon-3 / 8}<x^{7 / 8-\theta-3 \varepsilon}=x^{3 / 8-2 \varepsilon} / a,
$$

since $29 / 48<5 / 8$. We therefore can apply Buchstab to the right-hand side of (5.15) to obtain

$$
\sum_{q_{j}, r} S\left(\mathcal{A}_{r q_{1} \ldots q_{n}}, b a^{-1}\right)-\sum_{\substack{q_{j}, r \\ b a^{-1} \leq s<(2 P)^{1 / 2}}} S\left(\mathcal{A}_{r q_{1} \ldots q_{n} s}, s\right),
$$

where the first sum can be estimated by Lemma 13 . The second sum counts numbers $r q_{1} \ldots q_{n} s t \in \mathcal{A}$, where $P /(s e) \leq t<2 e P / s$. We note that $(2 P)^{1 / 2}$ $<b$, so we can apply Lemma 12 when $s \geq a$. If $s<a$ then

$$
P /(s e)>P /(a e)>a \text { and } 2 P e / s \leq 2 P e a / b<b,
$$

so $t \in[a, b]$ and thus Lemma 12 is applicable in this case also. We therefore obtain a suitable formula for both sums in (5.17), which establishes (5.13) as required.

Lemma 15. Let $x^{3 \varepsilon} P<\max \left(b^{2} / a, x^{7 / 8-\theta}\right), P>e v / b^{2}$ and $b a^{-1}<Q$ $\leq b$. Then

$$
\sum_{\substack{p \sim P \\ q \sim Q}} S\left(\mathcal{A}_{p q}, q\right)=\sum_{\substack{p \sim P \\ q \sim Q}} S\left(\mathcal{B}_{p q}, q\right)(1+O(\varepsilon)) .
$$

Proof. If $Q \geq a$ we can apply Lemma 12 so we henceforth suppose $Q<a$. We first consider the case

$$
x^{7 / 8-\theta}<x^{3 \varepsilon} P<b^{2} / a
$$

(so $\theta<29 / 48$ ). Here we work in a similar manner to Lemma 14 to obtain

$$
\begin{aligned}
\sum_{\substack{p \sim P \\
q \sim Q}} S\left(\mathcal{A}_{p q}, q\right) & =\sum_{\substack{q \sim Q \\
r}} S\left(\mathcal{A}_{q r},(2 P)^{1 / 2}\right) \\
& =\sum_{\substack{q \sim Q \\
r}} S\left(\mathcal{A}_{q r}, b a^{-1}\right)-\sum_{\substack{q \sim Q \\
r, b a^{-1} \leq s<(2 P)^{1 / 2}}} S\left(\mathcal{A}_{q r s}, s\right) .
\end{aligned}
$$


Now

$$
r \ll \frac{v}{P Q}<\frac{v x^{\theta-7 / 8+3 \varepsilon} a}{b}=x^{6 \theta+2 \varepsilon-27 / 8}<x^{7 / 8-\theta-3 \varepsilon}=\frac{x^{3 / 8-2 \varepsilon}}{a}
$$

since $29 / 48<17 / 28(=0.607 \ldots)$. As $Q<a$ we may therefore apply Lemma 13 to the first sum in (5.19). The second sum in (5.19) counts numbers $q r s t \in \mathcal{A}$. As in the proof of Lemma 14 we discover that one of $s, t$ must belong to the interval $[a, b]$.

We now assume that $x^{3 \varepsilon} P \leq x^{7 / 8-\theta}$. We apply Buchstab directly to the left-hand side of (5.18) to obtain

$$
\sum_{\substack{p \sim P \\ q \sim Q}} S\left(\mathcal{A}_{p q}, b a^{-1}\right)-\sum_{\substack{p \sim P \\ q \sim Q \\ b a^{-1} \leq r<q}} S\left(\mathcal{A}_{p q r}, r\right) .
$$

Lemma 13 can be applied to the first sum in (5.20). Now for the hypothesis of Lemma 15 to hold we must have $x^{7 / 8-\theta}>v / b^{2}$ so $\theta<39 / 64$. It then follows that $\left(b a^{-1}\right)^{2}>a$. We can therefore apply Lemma 12 to those parts of the second sum with $q r \leq b$. For the remaining portion of the sum we note that it counts numbers pqrs $\in \mathcal{A}$ where

$$
s<\frac{e v}{P q r} \leq \frac{e v}{\left(e v / b^{2}\right) b}=b,
$$

and

$$
s>\frac{v}{8 P Q^{2}} \geq \frac{v}{8 x^{7 / 8-\theta-3 \varepsilon} a^{2}}=\frac{x^{1 / 8+5 \varepsilon}}{8}>a .
$$

Hence Lemma 12 is again applicable and this completes the proof.

6. Implementing the alternative sieve. We would like to give an upper bound for $S\left(\mathcal{A}, v^{1 / 2}\right)$ of the form $u(\theta) y /(\theta \log x)$ where $u(\theta)$ is as small as possible. For $\theta<0.6$ we have obtained the "correct" value 1 . The method we now present gives a continuous function $u(\theta)$ starting with $u(0.6)=1$. Sadly, by the time $\theta$ reaches $39 / 64(=0.609 \ldots)$, the value of $u(\theta)$ is nearly 2. From this point onwards we are giving an upper bound for $S(\mathcal{A}, b)$. Although this upper bound is very close to the expected value of $S(\mathcal{A}, b)$, the substitution of $b$ for $v^{1 / 2}$ causes the marked deterioration in the quality of our bound. Nevertheless, our method here still produces a superior result to that obtained from the Rosser-Iwaniec sieve (see Lemma 17 below) up to about $\theta=0.661$. Theoretically the alternative sieve will be better up to $\theta=0.7$, but the calculations necessary become impractical. The values of $u(\theta)$ are plotted on Diagram 1 (see Section 7). 
We begin by using the Buchstab identity to write

$$
S\left(\mathcal{A}, v^{1 / 2}\right)=S\left(\mathcal{A}, b a^{-1}\right)-\sum_{b a^{-1} \leq p \leq b} S\left(\mathcal{A}_{p}, p\right)-\sum_{b<p<v^{1 / 2}} S\left(\mathcal{A}_{p}, p\right) .
$$

The contribution from the first two terms on the right-hand side of (6.1) equals $S(\mathcal{A}, b)$. We will only be able to give a non-trivial estimate for the final term when $\theta<39 / 64$. We start by giving a lower bound for this term. We write

$$
y=x^{-3 \varepsilon} \max \left(b^{2} / a, x^{7 / 8-\theta}\right) .
$$

Then, for $\theta<39 / 64$,

$$
\begin{aligned}
& \sum_{b<p<v^{1 / 2}} S\left(\mathcal{A}_{p}, p\right) \geq \sum_{e v / b^{2}<p<y} S\left(\mathcal{A}_{p}, p\right) \\
& =\sum_{e v / b^{2}<p<y} S\left(\mathcal{A}_{p}, b a^{-1}\right)-\sum_{\substack{e v / b^{2}<p<y \\
b a^{-1} \leq q<\min \left(p,(e v / p)^{1 / 2}\right)}} S\left(\mathcal{A}_{p q}, q\right) .
\end{aligned}
$$

We obtain an asymptotic formula for the first term in (6.2) from Lemma 14, and for the second term from Lemma 15 , since $p>e v / b^{2}$ gives $(e v / p)^{1 / 2}<b$. Thus

$$
\sum_{b<p<v^{1 / 2}} S\left(\mathcal{A}_{p}, p\right) \geq \sum_{e v / b^{2}<p<y} S\left(\mathcal{B}_{p}, p\right)(1+O(\varepsilon)) .
$$

Now we can apply Lemma 13 to $S\left(\mathcal{A}, b a^{-1}\right)$ in $(6.1)$, and Lemma 12 to

$$
-\sum_{a \leq p \leq b} S\left(\mathcal{A}_{p}, p\right)
$$

This leaves a term

$$
\begin{aligned}
(6.4)-\sum_{b a^{-1} \leq p<a} S\left(\mathcal{A}_{p}, p\right)= & -\sum_{b a^{-1} \leq p<a} S\left(\mathcal{A}_{p}, b a^{-1}\right)+\sum_{b a^{-1} \leq q<p<a} S\left(\mathcal{A}_{p q}, q\right) \\
= & -\sum_{b a^{-1} \leq p<a} S\left(\mathcal{A}_{p}, b a^{-1}\right)+\sum_{b a^{-1} \leq q<p<a} S\left(\mathcal{A}_{p q}, b a^{-1}\right) \\
& -\sum_{\substack{b a^{-1} \leq r<q<p<a \\
p q r^{2} \leq e v}} S\left(\mathcal{A}_{p q r}, r\right) \\
= & -\sum_{1}+\sum_{2}-\sum_{3} \text {, say. }
\end{aligned}
$$

We can apply Lemma 13 to $\sum_{1}$ and $\sum_{2}$ to obtain asymptotic formulae. We then want a lower bound for $\sum_{3}$. Clearly, if any product of 2 or 3 of $p, q, r$ lies in the interval $I$ we can apply Lemma 12 . Otherwise it may be possible to decompose the sums further. We require some notation to discuss these 
points. We write

$$
(p, q, r)=\left(x^{\alpha_{1}}, x^{\alpha_{2}}, x^{\alpha_{3}}\right)
$$

and similarly introduce $\alpha_{4}, \alpha_{5}, \ldots$ when performing further decompositions of $\sum_{3}$ as we bring in new prime variables $s=x^{\alpha_{4}}, t=x^{\alpha_{5}}, \ldots$ We put

$$
\begin{array}{r}
E_{n}=\left\{\boldsymbol{\alpha}=\left(\alpha_{1}, \ldots, \alpha_{n}\right) \in \mathbb{R}^{n}: g \leq \alpha_{n}<\alpha_{n-1}<\ldots<\alpha_{1}<\theta-1 / 2+\varepsilon,\right. \\
\left.\alpha_{1}+\ldots+\alpha_{n-1}+2 \alpha_{n} \leq \theta+1 / L\right\},
\end{array}
$$

where $g=g(\theta)$, as defined at the start of Section 4. The sum over $p, q, r$ in $\sum_{3}$ thus corresponds to $\boldsymbol{\alpha} \in E_{3}$. Given a set $Z \subset \mathbb{R}^{n}$ we let $Z^{\mathrm{c}}=\{\boldsymbol{\alpha} \in$ $\left.\mathbb{R}^{n}: \boldsymbol{\alpha} \notin Z\right\}$. A point $\boldsymbol{\alpha}$ of $E_{n}$ is said to be bad if

$$
\text { no } \operatorname{sum} \sum_{j \in \mathcal{S}} \alpha_{j}(\mathcal{S} \subset\{1, \ldots, n\}) \text { lies in }[\theta-1 / 2+\varepsilon, \tau(\theta)-\varepsilon] \text {. }
$$

The other points of $E_{n}$ are called good. These correspond to parts of sums for which Lemma 12 can be applied. We write $A_{n}$ for the set of bad points of $E_{n}$,

- $W=\left\{\left(\alpha_{1}, \alpha_{2}, \alpha_{3}\right) \in A_{3}\right.$ : either $\alpha_{2}+\alpha_{3}>7 / 8-\theta-4 \varepsilon$ or $\left(\alpha_{1}, \alpha_{2}, \alpha_{3}, \alpha_{4}\right) \in A_{4}$ for at least one $\alpha_{4} \in\left[g, \alpha_{3}\right)$ for which $\left.\alpha_{2}+\alpha_{3}+\alpha_{4} \geq 7 / 8-\theta-4 \varepsilon\right\}$,

- $U=A_{3} \cap W^{\mathrm{c}}, Z=E_{3} \cap A_{3}^{\mathrm{c}}$,

- $X_{1}=\left\{\left(\alpha_{1}, \alpha_{2}, \alpha_{3}, \alpha_{4}\right) \in E_{4} \cap A_{4}^{\mathrm{c}}:\left(\alpha_{1}, \alpha_{2}, \alpha_{3}\right) \in U\right\}$,

- $X_{2}=\left\{\left(\alpha_{1}, \alpha_{2}, \alpha_{3}, \alpha_{4}\right) \in A_{4}:\left(\alpha_{1}, \alpha_{2}, \alpha_{3}\right) \in U\right\}$

(we note that $\alpha_{2}+\alpha_{3}+\alpha_{4} \leq 7 / 8-\theta-4 \varepsilon$ in $X_{2}$ by the previous definitions),

- $V=\left\{\left(\alpha_{1}, \alpha_{2}, \alpha_{3}, \alpha_{4}, \alpha_{5}\right) \in A_{5}:\left(\alpha_{1}, \alpha_{2}, \alpha_{3}, \alpha_{4}\right) \in X_{2}\right\}$.

We first observe that $E_{3}$ partitions into $W, U$ and $Z$. Since all the points of $Z$ are good, we obtain the desired asymptotic formula for the part of $\sum_{3}$ with $\boldsymbol{\alpha} \in Z$ using Lemma 12. In contrast, the part with $\boldsymbol{\alpha} \in W$ must be discarded, that is, the trivial estimate

$$
\sum_{\mathrm{ff} \in W} S\left(\mathcal{A}_{p q r}, r\right) \geq 0
$$

appears to be the only one accessible with the available tools.

To see this, apply a further decomposition to any subset $W^{\prime}$ of $W$,

$$
\begin{aligned}
\sum_{\mathrm{ff} \in W^{\prime}} S\left(\mathcal{A}_{p q r}, r\right) & =\sum_{\mathrm{ff} \in W^{\prime}} S\left(\mathcal{A}_{p q r}, b a^{-1}\right)-\sum S\left(\mathcal{A}_{p q r s}, s\right) \\
& =\sum_{1}-\sum_{2}, \text { say. }
\end{aligned}
$$

In $\sum_{2},(p, q, r, s)=\left(x^{\alpha_{1}}, \ldots, x^{\alpha_{4}}\right)$,

$$
\left(\alpha_{1}, \alpha_{2}, \alpha_{3}\right) \in W^{\prime}, \quad \alpha_{4} \in\left[g, \alpha_{3}\right) .
$$


If $W^{\prime}$ overlaps the region

$$
\alpha_{2}+\alpha_{3}>7 / 8-\theta-4 \varepsilon,
$$

we cannot always split $p q r$ into two subproducts respectively less than $a$ and $x^{3 / 8-2 \varepsilon} /(2 a)$, so Lemma 13 will not handle $\sum_{1}$. Neither can we use Lemma 12 to handle $\sum_{1}$, by definition of $A_{3}$. If, on the other hand, $W^{\prime}$ does not overlap this half-space, it overlaps the set of $\left(\alpha_{1}, \alpha_{2}, \alpha_{3}\right)$ for which $\left(\alpha_{1}, \alpha_{2}, \alpha_{3}, \alpha_{4}\right) \in A_{4}$ for at least one $\alpha_{4} \in\left[g, \alpha_{3}\right)$, such that

$$
\alpha_{2}+\alpha_{3}+\alpha_{4}>7 / 8-\theta-4 \varepsilon \text {. }
$$

We cannot split $p, q, r, s$ into two subproducts which Lemma 13 will handle, so a further application of Buchstab to $\sum_{2}$ offers no way out; nor will Lemma 12 help, by definition of $A_{4}$.

We now turn our attention to the sum over $\boldsymbol{\alpha} \in U$. Applying Buchstab's identity we obtain

$$
\begin{aligned}
& -\sum_{\mathrm{ff} \in U} S\left(\mathcal{A}_{p q r}, r\right) \\
& =-\sum_{\mathrm{ff} \in U} S\left(\mathcal{A}_{p q r}, b a^{-1}\right)+\sum_{\mathrm{ff} \in X_{1}} S\left(\mathcal{A}_{p q r s}, s\right)+\sum_{\mathrm{ff} \in X_{2}} S\left(\mathcal{A}_{p q r s}, s\right) .
\end{aligned}
$$

The first term on the right side of (6.5) may be estimated by Lemma 13 since $\alpha_{2}+\alpha_{3} \leq 7 / 8-\theta-4 \varepsilon$ for all $\boldsymbol{\alpha} \in U$. The sum over $\boldsymbol{\alpha} \in X_{1}$ may be estimated by Lemma 12 since all $\boldsymbol{\alpha}$ are good in $X_{1}$. We apply Buchstab once more to the sum over $\boldsymbol{\alpha} \in X_{2}$ :

$$
\begin{aligned}
\sum_{\mathrm{ff} \in X_{2}} S\left(\mathcal{A}_{\text {pqrs }}, s\right) & \\
& =\sum_{\mathrm{ff} \in X_{2}} S\left(\mathcal{A}_{p q r s}, b a^{-1}\right)-\sum_{\substack{\mathrm{ff} \in E_{5} \\
\left(\alpha_{1}, \alpha_{2}, \alpha_{3}, \alpha_{4}\right) \in X_{2}}} S\left(\mathcal{A}_{\text {pqrst }}, t\right) .
\end{aligned}
$$

We can apply Lemma 13 to the first sum on the right side of (6.6) since $\alpha_{2}+\alpha_{3}+\alpha_{4} \leq 7 / 8-\theta-4 \varepsilon$ in $X_{2}$, and we can apply Lemma 12 for all the good $\boldsymbol{\alpha}$ in the final sum. This leaves a final sum to consider:

$$
-\sum_{\mathrm{ff} \in V} S\left(\mathcal{A}_{p q r s t}, t\right)
$$

We are unable to give a formula for any part of this sum without further applications of Buchstab's identity. Discarding this sum would give rise to a loss of

$$
\frac{y}{L} \int_{V} w\left(\frac{\theta-\alpha_{1}-\alpha_{2}-\alpha_{3}-\alpha_{4}-\alpha_{5}}{\alpha_{5}}\right) \frac{d \alpha_{1}}{\alpha_{1}} \ldots \frac{d \alpha_{4}}{\alpha_{4}} \frac{d \alpha_{5}}{\alpha_{5}^{2}},
$$


after using the standard procedure for replacing sums over primes by integrals (cf. [1], p. 227).

To explain what is meant by a "loss" of the quantity (6.8), we illustrate by a simpler example. From (6.1),

$$
S\left(\mathcal{A}, v^{1 / 2}\right)=S\left(\mathcal{A}, b a^{-1}\right)-\sum_{a \leq p \leq b} S\left(\mathcal{A}_{p}, p\right)-\sum_{p \in P} S\left(\mathcal{A}_{p}, p\right),
$$

where $P=\left[b a^{-1}, a\right) \cup\left(b, v^{1 / 2}\right)$. Suppose we were to discard the last sum. Since

$$
S\left(\mathcal{B}, v^{1 / 2}\right)=S\left(\mathcal{B}, b a^{-1}\right)-\sum_{a \leq p \leq b} S\left(\mathcal{B}_{p}, p\right)-\sum_{p \in P} S\left(\mathcal{B}_{p}, p\right),
$$

and since we have asymptotic formulae for what we do not discard, we would find that

$$
\begin{aligned}
S\left(\mathcal{A}, v^{1 / 2}\right) & \lesssim S\left(\mathcal{B}, v^{1 / 2}\right)+\sum_{p \in P} S\left(\mathcal{B}_{p}, p\right) \\
& \lesssim S\left(\mathcal{B}, v^{1 / 2}\right)+\frac{y}{L} \int_{P^{\prime}} w\left(\frac{\theta-\alpha_{1}}{\alpha_{1}}\right) \frac{d \alpha_{1}}{\alpha_{1}^{2}} .
\end{aligned}
$$

Here $P^{\prime}=[5 / 2-4 \theta, \theta-1 / 2] \cup[2-3 \theta, \theta / 2]$. Our "loss" by this crude procedure would be the integral over $P^{\prime}$. Using instead the approach above, which results in discarding

$$
P^{\prime \prime}=[2-3 \theta, 7 \theta-4] \cup\left[\max \left(\frac{9-14 \theta}{2}, \frac{7-8 \theta}{8}\right), \frac{\theta}{2}\right]
$$

in $\mathbb{R}^{1}, W$ in $\mathbb{R}^{3}$ and $V$ in $\mathbb{R}^{5}$, we are led by a similar argument to a loss of

$$
\int_{P^{\prime \prime}} w\left(\frac{\theta-\alpha_{1}}{\alpha_{1}}\right) \frac{d \alpha_{1}}{\alpha_{1}^{2}}+K(\theta)+R^{\prime}(\theta),
$$

where

$$
\begin{gathered}
K(\theta)=\int_{W} w\left(\frac{\theta-\alpha_{1}-\alpha_{2}-\alpha_{3}}{\alpha_{3}}\right) \frac{d \alpha_{1} d \alpha_{2} d \alpha_{3}}{\alpha_{1} \alpha_{2} \alpha_{3}^{2}} \\
R^{\prime}(\theta)=\int_{V} w\left(\frac{\theta-\alpha_{1}-\alpha_{2}-\alpha_{3}-\alpha_{4}-\alpha_{5}}{\alpha_{5}}\right) \frac{d \alpha_{1} d \alpha_{2} d \alpha_{3} d \alpha_{4} d \alpha_{5}}{\alpha_{1} \alpha_{2} \alpha_{3} \alpha_{4} \alpha_{5}^{2}} .
\end{gathered}
$$

Since $u w(u)=1$ for $1 \leq u \leq 2$, and

$$
u w(u)=1+\log (u-1) \quad(2 \leq u \leq 3),
$$

a straightforward calculation now leads to

$$
S\left(\mathcal{A}, v^{1 / 2}\right) \lesssim \frac{y}{L}\left(M(\theta)+K(\theta)+R^{\prime}(\theta)\right) .
$$


Here

$$
M(\theta)=\frac{1}{\theta}\left(1+\log \left(\frac{7 \theta-4}{2-3 \theta} \cdot \frac{16 \theta-9}{9-14 \theta}\right)+\int_{2}^{\beta(\theta)} \frac{1+\log (v-1)}{v} d v\right)
$$

for $3 / 5-\varepsilon<\theta<29 / 48$;

$$
M(\theta)=\frac{1}{\theta}\left(1+\log \left(\frac{7 \theta-4}{2-3 \theta} \cdot \frac{16 \theta-7}{7-8 \theta}\right)+\int_{2}^{\beta(\theta)} \frac{1+\log (v-1)}{v} d v\right)
$$

for $29 / 48 \leq \theta<39 / 64$;

$$
M(\theta)=\frac{1}{\theta}\left(1+\log 2+\int_{2}^{\beta(\theta)} \frac{1+\log (v-1)}{v} d v\right)
$$

for $\theta \geq 39 / 64$.

In $(6.11)-(6.13), \beta(\theta)=\theta / \tau(\theta)-1$. In $(6.13)$,

$$
M(\theta)=\frac{1}{\tau(\theta)} w\left(\frac{\theta}{\tau(\theta)}\right) \quad \text { since } \frac{\theta}{\tau(\theta)} \in[3,4] .
$$

We can improve on (6.10), although the improvement only becomes significant for $\theta \in[0.64,0.661]$. Let $V_{1}$ be the subset of $V$ for which the sum $\alpha_{1}+\alpha_{2}+\alpha_{3}+\alpha_{4}+\alpha_{5}+\alpha_{5}$ decomposes into two subsums less than $\theta-1 / 2-2 \varepsilon$ and $7 / 8-\theta-4 \varepsilon$; for instance, $\boldsymbol{\alpha} \in V_{1}$ whenever $\boldsymbol{\alpha} \in V$ and

$$
\alpha_{1}+\alpha_{2}+\alpha_{3}+\alpha_{4}+2 \alpha_{5}<7 / 8-\theta-4 \varepsilon .
$$

For the subsum of (6.7) with $\boldsymbol{\alpha} \in V_{1}$, two more applications of Buchstab may be handled in essentially the same way as (6.6). We do not extract all the information this yields, owing to the large number of ways seven variables might combine to give a value in $[\theta-1 / 2+\varepsilon, \tau(\theta)-\varepsilon]$. However,

$$
\begin{aligned}
\int_{g}^{\alpha_{5}} \int_{g}^{\alpha_{6}} w\left(\frac{\theta-\alpha_{1}-\ldots-\alpha_{7}}{\alpha_{7}}\right) \frac{d \alpha_{6} d \alpha_{7}}{\alpha_{6} \alpha_{7}^{2}} & <0.57 \int_{g}^{\alpha_{5}} \int_{g}^{\alpha_{6}} \frac{d \alpha_{6}}{\alpha_{6}} \frac{d \alpha_{7}}{\alpha_{7}^{2}} \\
& =0.57\left(\frac{1}{g} \log \left(\frac{\alpha_{5}}{g}\right)-\frac{1}{g}+\frac{1}{\alpha_{5}}\right)
\end{aligned}
$$

for $\boldsymbol{\alpha} \in V$, since one may verify that $\theta-\alpha_{1}-\ldots-\alpha_{7}>3 \alpha_{7}$. (For the bound $w(u) \leq 0.57$ for $u \geq 3$, see [4].)

Let $V_{2}$ be that part of $V_{1}$ where

$$
0.57\left(\frac{1}{g} \log \left(\frac{\alpha_{5}}{g}\right)-\frac{1}{g}+\frac{1}{\alpha_{5}}\right)<\frac{1}{\alpha_{5}} w\left(\frac{\theta-\alpha_{1}-\ldots-\alpha_{5}}{\alpha_{5}}\right)
$$

and $V_{3}$ the complement of $V_{2}$ in $V$. Our discussion shows that in (6.10), 
$R^{\prime}(\theta)$ may be replaced by

$$
\begin{aligned}
R(\theta)= & \frac{y}{L}\left(\int_{V_{3}} w\left(\frac{\theta-\alpha_{1}-\ldots-\alpha_{5}}{\alpha_{5}}\right) \frac{d \alpha_{1} \ldots d \alpha_{5}}{\alpha_{1} \ldots \alpha_{4} \alpha_{5}^{2}}\right. \\
& \left.+0.57 \int_{V_{2}}\left(\frac{1}{g} \log \frac{\alpha_{5}}{g}-\frac{1}{g}+\frac{1}{\alpha_{5}}\right) \frac{d \alpha_{1}}{\alpha_{1}} \ldots \frac{d \alpha_{5}}{\alpha_{5}}\right) .
\end{aligned}
$$

We record this conclusion in a lemma.

LEMma 16. For $3 / 5-\varepsilon<\theta \leq 0.661$ we have

$$
S\left(\mathcal{A}, v^{1 / 2}\right) \lesssim \frac{y}{L}(M(\theta)+K(\theta)+R(\theta)) .
$$

Here $M(\theta), K(\theta), R(\theta)$ are defined by (6.11)-(6.13), (6.9) and (6.14).

We have tacitly assumed so far that it will always be to our advantage to effect the first five decompositions when these are possible. For $\theta \geq 0.65$ this is not always the case. To allow for this, we define

$$
I_{1}\left(\alpha_{1}, \alpha_{2}, \alpha_{3}\right)=\int_{\left(\alpha_{1}, \ldots, \alpha_{5}\right) \in V}^{*} w\left(\frac{\theta-\alpha_{1}-\ldots-\alpha_{5}}{\alpha_{5}}\right) \frac{d \alpha_{4}}{\alpha_{4}} \frac{d \alpha_{5}}{\alpha_{5}^{2}}
$$

where the $*$ indicates that the integral is to be subdivided further as in (6.14). Now let

$$
\begin{gathered}
I_{2}\left(\alpha_{1}\right) \\
=\int_{\left(\alpha_{1}, \alpha_{2}, \alpha_{3}\right) \in U} \min \left(\frac{1}{\alpha_{3}} w\left(\frac{\theta-\alpha_{1}-\alpha_{2}-\alpha_{3}}{\alpha_{3}}\right), I_{1}\left(\alpha_{1}, \alpha_{2}, \alpha_{3}\right)\right) \frac{d \alpha_{2}}{\alpha_{2}} \frac{d \alpha_{3}}{\alpha_{3}}, \\
I_{3}\left(\alpha_{1}\right)=\int_{\left(\alpha_{1}, \alpha_{2}, \alpha_{3}\right) \in W} w\left(\frac{\theta-\alpha_{1}-\alpha_{2}-\alpha_{3}}{\alpha_{3}}\right) \frac{d \alpha_{2}}{\alpha_{2}} \frac{d \alpha_{3}}{\alpha_{3}^{2}}
\end{gathered}
$$

and

$$
I_{4}(\theta)=\int_{g}^{\theta-1 / 2} \min \left(\frac{1}{\alpha_{1}} w\left(\frac{\theta-\alpha_{1}}{\alpha_{1}}\right), I_{2}\left(\alpha_{1}\right)+I_{3}\left(\alpha_{1}\right)\right) \frac{d \alpha_{1}}{\alpha_{1}} .
$$

A very slight improvement in Lemma 16 is attained by replacing $K(\theta)+R(\theta)$ by $I_{4}(\theta)$.

We finish this section by revisiting the bounds obtained from the RosserIwaniec sieve. We can combine Lemma 7 with Lemma 12 to obtain the following result.

Lemma 17. For $\theta \in(0.661,0.7]$, we have

$$
L y^{-1} S(\theta) \lesssim \frac{2}{\varrho(\theta)}-\int_{\theta-1 / 2}^{\tau(\theta)} w\left(\frac{\theta-\alpha}{\alpha}\right) \frac{d \alpha}{\alpha^{2}}-\int_{B} w\left(\frac{\theta-\alpha_{1}-\alpha_{2}}{\alpha_{2}}\right) \frac{d \alpha_{1} d \alpha_{2}}{\alpha_{1} \alpha_{2}^{2}}
$$


Here

$$
B=\left\{\left(\alpha_{1}, \alpha_{2}\right): \varrho(\theta) / 3<\alpha_{1}<\theta-1 / 2, \theta-1 / 2<\alpha_{2}<\tau(\theta)\right\} .
$$

Proof. We note that $S(\mathcal{A}, z)$ counts many numbers not counted by $S(\theta)$. For some of these we can apply Lemma 12 and so obtain an improved bound by removing the "deductible" terms. To be precise, we have

$$
S(\theta) \leq S(\mathcal{A}, z)-\sum_{\substack{p m \in \mathcal{A} \\
p \in I \\
Q(m)>p}} 1-\sum_{\begin{array}{c}
p_{1} p_{2} m \in \mathcal{A} \\
z<p_{1}<a, p_{2} \in I \\
Q(m)>p_{2}
\end{array}} 1 .
$$

We note that for the values of $\theta$ in the lemma we have $z<a$ and so the deductible sums are non-empty. Since Lemma 12 can be applied to both of these sums we can replace sums by integrals in (6.15) and use Lemma 7 for $S(\mathcal{A}, z)$ to complete the proof.

7. Completion of the proof. The graph of our upper bound for $\theta S(\theta) L y^{-1}$ is shown in Diagram 1 (as $u(\theta)$ ).

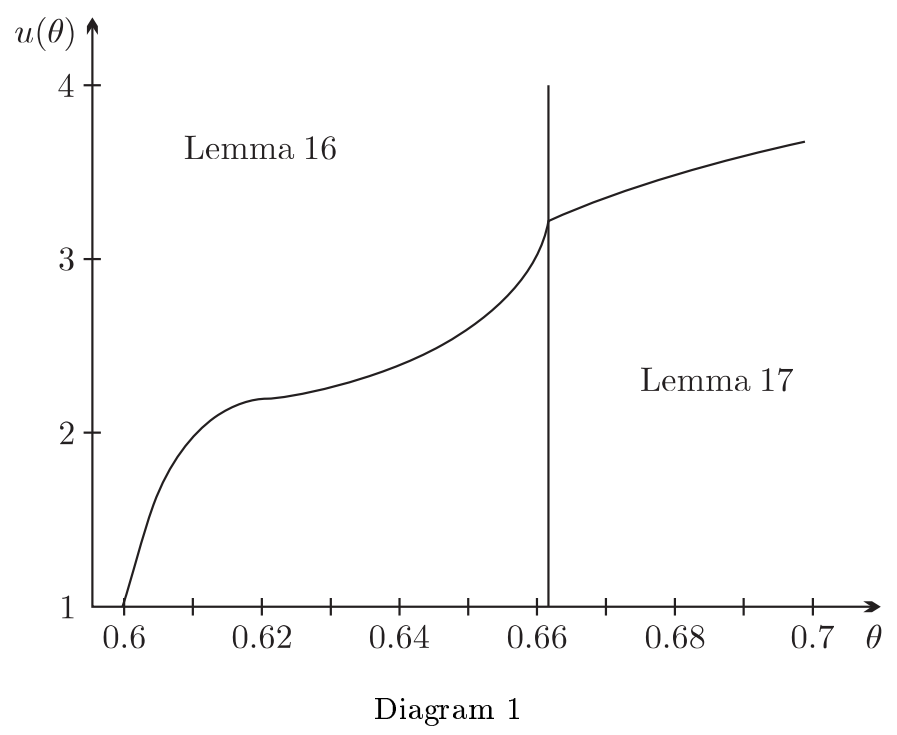

From Lemma 17 we obtain

$$
\int_{0.661}^{0.7} \theta S(\theta) d \theta<0.1386 y L^{-1}
$$

Using Lemma 16 we note that

$$
\int_{0.6}^{0.661} \theta M(\theta) d \theta<0.1256
$$


and

$$
\int_{0.6}^{0.661} \theta \min \left(I_{4}(\theta), K(\theta)+R(\theta)\right) d \theta<0.0125 .
$$

We remark that it is straightforward to obtain very accurate estimates for the integrals in (7.1) and (7.2). The estimate for the integral in (7.3) is an upper bound, but a more precise estimate is more difficult to achieve. We thus obtain

$$
\int_{0.6}^{0.7} \theta S(\theta) d \theta<0.2767 y L^{-1}
$$

Since, by Lemma 7 ,

$$
\frac{L}{y} \int_{0.7}^{\beta} \theta S(\theta) d \theta \leq \frac{16}{3} \int_{0.7}^{\beta} \theta d \theta=\frac{8}{3}\left(\beta^{2}-0.49\right),
$$

this indicates that our theorem holds for any exponent less than

$$
\left(0.49+\frac{3}{8}(0.4-0.2767)\right)^{1 / 2}>0.732
$$

which establishes Theorem 1.

We finish by remarking that it appears to be very difficult to make any further progress without new exponential sum estimates. To increase the exponent just by 0.001 would require us to make a saving of nearly 0.004 between 0.6 and 0.7 . The only room for improvement seems to be in $K(\theta)+R(\theta)$ for $\theta \geq 0.64$. Even at $\theta=0.64$, we have $\theta(K(\theta)+R(\theta))<0.42$. Assuming that we will have to switch to the method of Section 4 by $\theta=0.67$, it appears unlikely that we can make the necessary average saving of 0.13 between 0.64 and 0.67 without a new idea.

\section{References}

[1] R. C. Baker, The greatest prime factor of the integers in an interval, Acta Arith. 47 (1986), 193-231.

[2] R. C. Baker, G. Harman and J. Rivat, Primes of the form $\left[n^{c}\right]$, J. Number Theory, to appear.

[3] E. Bombieri and H. Iwaniec, On the order of $\zeta\left(\frac{1}{2}+i t\right)$, Ann. Scuola Norm. Sup. Pisa 13 (1986), 449-472.

[4] A. Y. Cheer and D. A. Goldston, A differential delay equation arising from the sieve of Eratosthenes, Math. Comp. 55 (1990), 129-141.

[5] H. Daven port, Multiplicative Number Theory, 2nd ed. revised by H. L. Montgomery, Springer, New York, 1980.

[6] E. Fouvry, Sur le théorème de Brun-Titchmarsh, Acta Arith. 43 (1984), 417-424. 
[7] E. Fouvry and H. Iwaniec, Exponential sums with monomials, J. Number Theory 33 (1989), 311-333.

[8] J. B. Friedlander, Integers free from large and small primes, Proc. London Math. Soc. 33 (1986), 565-576.

[9] S. W. Graham, The greatest prime factor of the integers in an interval, J. London Math. Soc. 24 (1981), 427-440.

[10] S. W. Graham and G. Kolesnik, Van der Corput's Method of Exponential Sums, Cambridge Univ. Press, 1991.

[11] G. Harman, On the distribution of $\alpha p$ modulo one, J. London Math. Soc. 27 (2) (1983), 9-13.

[12] C. H. Jia, The greatest prime factor of integers in short intervals II, Acta Math. Sinica 32 (1989), 188-199 (in Chinese).

[13] H.-Q. Liu, The greatest prime factor of the integers in an interval, Acta Arith. 65 (1993), 301-328.

[14] S. H. Min, Methods in Number Theory, Vol. 2, Science Press, 1983 (in Chinese).

[15] K. Ramachandra, A note on numbers with a large prime factor, J. London Math. Soc. 1 (2) (1969), 303-306.

[16] - A note on numbers with a large prime factor, II, J. Indian Math. Soc. 34 (1970), 39-48.

[17] E. C. Titchmarsh, The Theory of the Riemann Zeta-Function, revised by D. R. Heath-Brown, Oxford University Press, 1986.

[18] I. M. Vinogradov, The Method of Trigonometrical Sums in the Theory of Numbers, translated and annotated by A. Davenport and K. F. Roth, Wiley, New York, 1954.

[19] N. Watt, Exponential sums and the Riemann zeta function II, J. London Math. Soc. 39 (1989), 385-404.

[20] J. Wu, $P_{2}$ dans les petits intervalles, in: Séminaire de Théorie des Nombres, Paris 1989-90, Birkhäuser, 1992, 233-267.

DEPARTMENT OF MATHEMATICS

BRIGHAM YOUNG UNIVERSITY

PROVO, UTAH 84602

U.S.A.
MATHEMATICS INSTITUTE UNIVERSITY OF WALES SENGHENNYDD ROAD CARDIFF CF 2 4AG, U.K.

Received on 1.8.1994

and in revised form on 21.2.1995 